\title{
Graph Laplacian for Spectral Clustering and Seeded Image Segmentation
}

\author{
Wallace Casaca $^{1}$, Gabriel Taubin ${ }^{2}$ (co-advisor), Luis Gustavo Nonato ${ }^{1}$ (advisor) \\ ${ }^{1}$ Instituto de Ciências Matemáticas e de Computação (ICMC) - USP, São Carlos, Brasil \\ ${ }^{2}$ School of Engineering - Brown University, Box D, Providence, 02912, USA
}

\begin{abstract}
Interactive segmentation methods have gained much attention lately, specially due to their good performance in segmenting complex images and easy utilization. However, most interactive segmentation algorithms rely on sophisticated mathematical formulations whose effectiveness highly depends on the kind of image to be processed. In fact, sharp adherence to the contours of image segments, uniqueness of solution, high computational burden, and extensive user interaction are some of the weaknesses of most existing methods. In this thesis we proposed two novel interactive image segmentation techniques that sort out the issues raised above. The proposed methods rely on Laplace operators, spectral graph theory, and optimization schemes towards enabling highly accurate segmentation tools which demand a reduced amount of user interaction while still being mathematically simple and computationally efficient. The good performance of our segmentation algorithms is attested by a comprehensive set of comparisons against representative state-of-the-art methods. As additional contribution, we have also proposed two new algorithms for inpainting and photo colorization, both of which rely on the accuracy of our segmentation apparatus.
\end{abstract}

\section{Introduction}

Image segmentation is key task for an enormous quantity of computer vision problems. A typical procedure in image segmentation is to interpret an image as a graph, what enables the use of powerful mathematical tools such as Laplace operators and spectral graph theory in the context of segmentation. Moreover, the flexibility introduced by a graph representation as to pixel connectivity and edge weighting greatly increases the capability of segmentation algorithms to distinguish patterns, structures, and shapes. However, outperforming human skills in terms of pattern recognition is a difficult task. Therefore, semi-supervised image segmentation methods have become a trend by combining the human ability for patter recognition with the solid mathematical foundation of graph theory [Chung 1997]. In this context, the use of interact mechanisms to properly settle Laplacian operators on image graph representations have proven to be an effective alternative [Casaca et al. 2013b, Casaca et al. 2014b]. Those user-assisted mechanisms typically define the Laplacian operators in a similarity/affinity graph which encodes image information such as colors, textures and gradients. The segmentation is accomplished by either optimizing a cost function defined on the graph [Grady 2006, Casaca et al. 2014b] or by solving a spectral-cut problem [Shi and Malik 2000, Casaca et al. 2013b]. Despite their pliability and powerful, most state-of-the-art methods often result in segmentations with low adherence to the contours of the image segments, failing to capture fine details and thus producing a low quality segmentation [Peng and Zhang 2013]. Moreover, most existing techniques rely on sophisticated optimization resources that demands high computational effort while being highly sensitive to edge weights and user interaction. 


\subsection{Contributions}

In this thesis we proposed two novel user-assisted image segmentation techniques that sort out the issues discussed above. The proposed methods rely on Laplace operators, spectral graph theory, and optimization schemes towards enabling highly accurate segmentation tools which demand a reduced amount of user interaction while still being mathematically simple and computationally efficient. While most of our research has been focused on the particular problem of image segmentation, we develop as side results new methodologies for the problem of image inpainting [Casaca et al. 2014a] and photo colorization [Casaca et al. 2012], both of which derived from the proposed segmentation approaches. A summary of the main results obtained during the development of this thesis is presented in the following sections.

\section{Spectral Image Segmentation}

This proposed method derives from the so-called spectral clustering methods (reported in [Casaca 2014, Casaca et al. 2013b]) and it combines cartoon-texture decomposition [Casaca and Boaventura 2010], similarity metrics [Casaca et al. 2013b], and spectral graph theory [Chung 1997] into a unified framework (see Figure 1). The proposed approach holds attractive properties such as awareness to noise and texture, accuracy in detecting image edges, low computational cost and a reduced number of human intervention (see Figure 2 for a few illustrations). Qualitative and quantitative results are described in [Casaca 2014] and clearly show the effectiveness of our methodology, outperforming other spectral-cut based methods in terms of accuracy and smoothness.

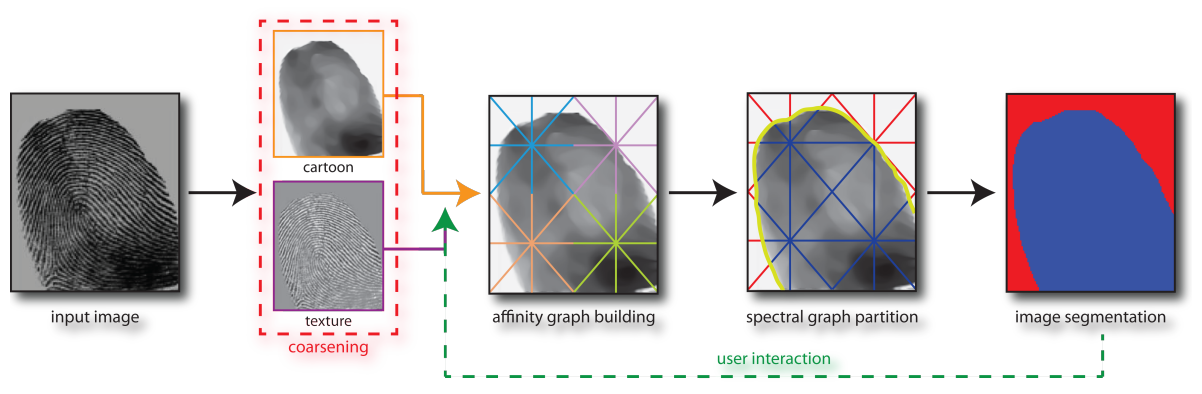

Figure 1. Pipeline of our spectral segmentation approach.
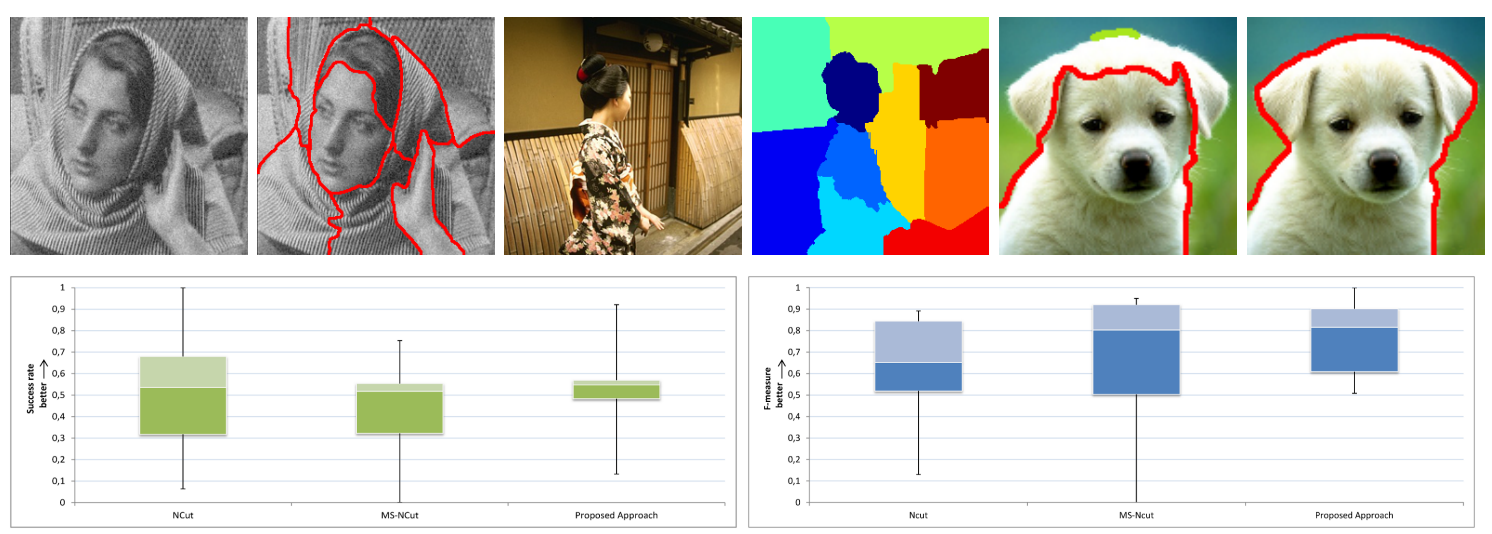

Figure 2. 1st row: Segmentation of a noise-and-textured image, example of multiple fragmentation, and illustration of the user interaction to improve the partition. 2nd row: Quantitative comparison (Success rate and F-measure) against NCut [Shi and Malik 2000] and MS-NCut [Cour et al. 2005]. 


\section{Seeded Image Segmentation}

We also propose a novel seed-based image segmentation technique that bears traits not present in other existing techniques. In contrast to most seed-based methods, our approach relies on a simple mathematical formulation that guarantees uniqueness of solution for the segmentation, presenting anisotropic behavior to ensure contour adherence. Moreover, our technique is not prone to be trapped in local minima while still demanding a few user interventions to work properly.

Laplacian Coordinates Energy Minimization on Graphs. As a basic tool to compute the Laplacian Coordinates (LC) energy, we define a weighted graph $G=\left(V, E, W_{E}\right)$ as in [Casaca et al. 2014b]. The Laplacian Coordinates energy $E$ is then computed as follows:

$$
\sum_{i \in B}\left\|x_{i}-x_{B}\right\|_{2}^{2}+\sum_{i \in F}\left\|x_{i}-x_{F}\right\|_{2}^{2}+\frac{1}{2} \sum_{i \sim j}\left\|w_{i j}\left(x_{i}-x_{j}\right)\right\|_{2}^{2},
$$

where $\mathbf{x}=\left(x_{1}, x_{2}, \ldots, x_{n}\right)$ is the saliency map which assigns a scalar value $x_{i}$ to each pixel $p_{i}$ of the image. The rationale behind the LC approach is that the non-pairwise terms from Equation (1) enforce fidelity of brushed pixels $p_{i}, i \in B \cup F$, to the scalars $x_{B}$ (background) and $x_{F}$ (foreground), while the last term imposes spatial smoothness within image segments and allows sharp jumps across image boundaries. Energy (1) is efficiently computed by solving a sparse system of linear equations, as outlined in [Casaca 2014].

Illustrative Results. As shown in [Casaca 2014, Casaca et al. 2014b], the proposed approach outperforms competing techniques quantitatively as well as qualitatively (see Fig. 3 for illustrative results). For applications using the proposed approach, see video ${ }^{1}$.

\footnotetext{
${ }^{1}$ https://www.youtube.com/watch?v=IbhxpmtFa0Q
}
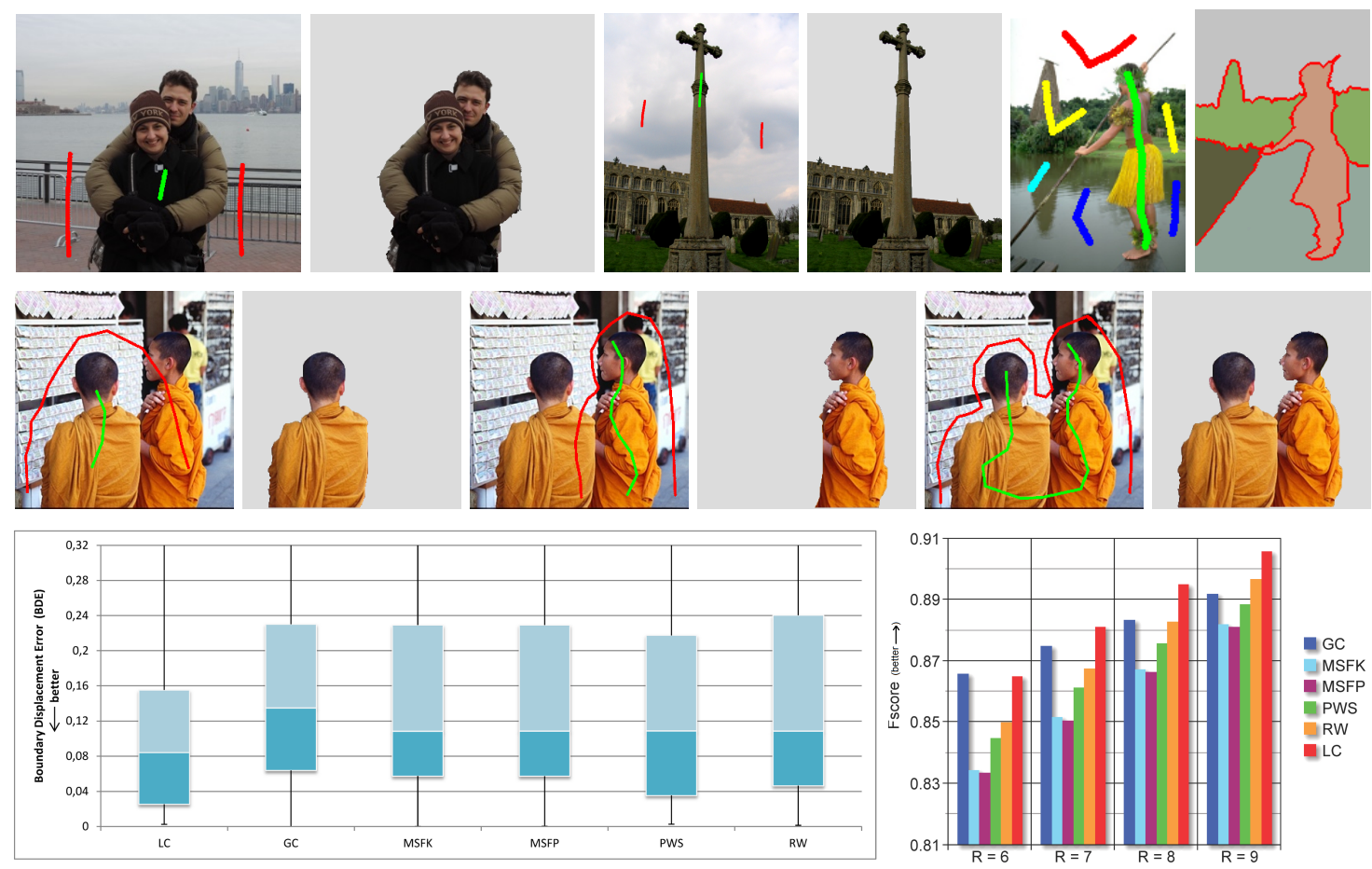

Figure 3. 1st row: segmentations produced by the proposed approach. 2nd row: flexibility in generating multiple partitions. 3rd row: comparison of our method (LC) against Graph Cuts (GC), Maximum Spanning Forest with Kruskal and Prim's algorithms (MSFK, MSFP), Power Watershed (PWS) and Random Walker (RW) [Grady 2006, Cousty et al. 2009, Couprie et al. 2011] for BDE and F-score. 


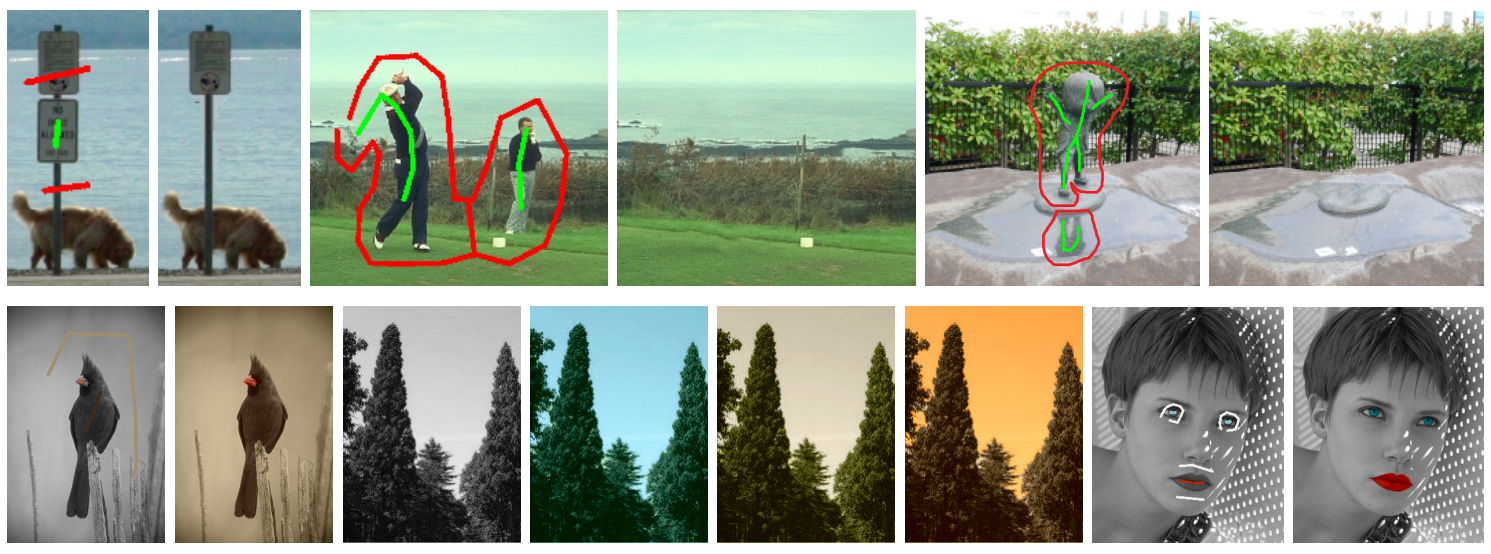

Figure 4. Image inpainting and colorization examples obtained from the proposed algorithms.

\section{Image Inpainting and Photo Colorization}

As additional contribution in the thesis [Casaca 2014], we also propose new algorithms for image inpainting and photo colorization (see Figure 4 and the video ${ }^{2}$ for illustrations). The methods rely on the accuracy of the proposed segmentation approaches to properly perform inpainting and image colorization, as reported in [Casaca 2014, Casaca et al. 2015c, Casaca et al. 2015a].

\section{Awards, Publications and Work Impact to the General Public}

\subsection{Awards}

The following papers have been awarded as "best papers" or received "honorable mention" in the renowned SIBGRAPI and ICCP conferences. According to Qualis-CAPES and Google Scholar, SIBGRAPI (Conference on Graphics, Patterns and Images) is the most relevant Latin American conference in the fields of Computer Graphics, Visualization, Patterns and Computer Vision. ICCP (IEEE International Conference on Computational Photography) is a prominent conference in the field of Digital Photography.

- Best paper award for the paper "Spectral Segmentation using Cartoon-Texture Decomposition and Inner Product-based Metric", 24th SIBGRAPI (IEEE), Maceio, 2011.

- Best paper award for the paper "Mixed Integer Optimization for Layout Arrangement", 26th SIBGRAPI (IEEE Computer Society), Arequipa, Peru, $2013^{3}$.

- Honorable Mention for the poster presentation "Image Colorization based on Multidimensional Projection”, 5th IEEE ICCP, Harvard, Cambridge, United States, 2014.

\subsection{Publications}

In the following we provide a summary of main publications originated during the $\mathrm{PhD}$ research. Qualis-CAPES as well as Google h5-index metric were also listed with the publications. According to Google Scholar, IEEE CVPR Conference figures at 1st among the most relevant publication venues in the general field of Computer Sciences, and it also ranks 7 th when taking into account all the Engineering fields. Finally, according to Microsoft Academic Research ${ }^{4}$, CVPR figures at the top of the ranking.

\footnotetext{
${ }^{2}$ https://www.youtube.com/watch?v=yrSIbP8IBr0

${ }^{3}$ See the video of our tool at https://www.youtube.com/watch?v=zGgIYX7oSqI

${ }^{4}$ Google Scholar: http://icmc.usp.br/e/aec2d, Microsoft Academic Research: http://icmc.usp.br/e/e95a9
} 


\section{Contributions Directly Related to the Thesis}

- [Casaca et al. 2011b] "Spectral Segmentation using Cartoon-Texture and Inner Productbased Metric", 24th SIBGRAPI, IEEE Computer Society (Qualis B1, h5-index: 12).

- [Casaca et al. 2012] "Colorization by Multidimensional Projection", 25th Conference on Graphics, Patterns and Images, IEEE Computer Society (Qualis B1, h5-index: 12).

- [Casaca et al. 2013b] "Image Segmentation using Image Decomposition and Inner Product based Metric", Journal of Math. Imaging and Vision (Qualis B1, h5-Index: 27).

- [Casaca et al. 2014a] "Comb. Anisotropic Diffusion, Transport Eq. and Texture Synthesis for Inpainting Text. Images", Pattern Recognition Letters (Qualis A1, h5-Index: 44).

- [Casaca et al. 2014b] "Laplacian Coordinates for Seeded Image Segmentation", IEEE Conf. Computer Vision and Pattern Recognition (CVPR) (Qualis A1, h5-Index: 118).

- [Casaca et al. 2015a] Interac. Image Colorization using Laplacian Coordinates, 16th Int. Conf. on Computer Analysis of Images and Patterns (CAIP) (Qualis B1, h5-Index: 16).

- [Casaca et al. 2015c] "A User Interact. Image Inpainting Framework using Laplacian Coord.", IEEE International Conf. on Image Processing (ICIP) (Qualis A1, h5-Index: 35).

\section{Other Publications}

- [Casaca et al. 2011a] "Classifying Texture and Free-Textured Content from Images using Nonlinear Anisotropic Diffusion", 5th Conference on Mathematical Analysis.

- [Joia et al. 2012] "Class-specific Metrics for Multidimensional Data Projection applied to CBIR”, Visual Computer (Qualis B1, h5-Index: 20).

- [Casaca et al. 2013a] "Denoising Textured Images via Regularized Anisotropic Diffusion" (Chapter Book, An Introductory Guide to Image and Video Processing).

- [Gomez-Nieto et al. 2013] "Mixed Integer Optimization for Layout Arrangement", 26th SIBGRAPI, IEEE Computer Society (Qualis B1, h5-Index: 12).

- [Gomez-Nieto et al. 2014] "Similarity Preserving Snippet-Based Vis. of Web Search Results", IEEE Trans. Visualization and Computer Graphics (Qualis A1, h5-Index: 50).

- [Casaca et al. 2015b] "Dealing with Multiple Requirements in Geometric Arrangements", IEEE Trans. Visualization and Computer Graphics (TVCG) (Qualis A1, h5-Index: 50).

\section{Papers under Review}

- Casaca, W., Nonato, L.G., Taubin, G., Laplacian Coordinates for Interactive Image Segmentation, IEEE Trans. on PAMI (CVPR's paper extension) (Qualis A1, h5-Index: 104).

- Motta, D., Casaca, W., Gomez-Nieto, E., Paiva, A., Identifying Blood Vessels Changes in Series of Retinal Fundus Image, Neurocomputing (Qualis A1, h5-Index: 45).

\subsection{Scientific Dissemination to the General Public}

The research conducted during the thesis had a good impact in terms of diffusion to the general public. In fact, part of our research has been advertised in TV news channels, news papers, and in the internet, as listed below:

1. Report published on www.usp.br (2014): "ICMC desenvolve ferramenta inovadora para segmentação de imagens" (www. icmc.usp.br/e/f37b3).

2. Report published on DCI (Newspaper) (2014): "Pesquisadores desenvolvem nova ferramenta para imagem" (www. icmc.usp.br/e/0ebf1).

3. TV report broadcasted on Rede Globo (2014): "Programa criado pela USP São Carlos remove pessoas de foto de forma fácil" (www. icmc.usp.br/e/49c86).

4. TV report broadcasted on TV Educativa de São Carlos (2014): "USP cria ferramenta que facilita a vida de quem trabalha com imagens" (www. icmc. usp.br/e/9daca). 


\section{References}

Casaca, W. (2014). Graph Laplacian for Spectral Clustering and Seeded Image Segmentation. 161 pgs, PhD Thesis, University of São Paulo, São Carlos, Brazil.

Casaca, W., Almeida, M. P., and Boaventura, M. (2013a). Denoising textured images via regularized anisotropic diffusion. In An Introduc. Guide to Image and Video Proc., pages 48-71. Brisbane, Australia.

Casaca, W., Almeida, M. P., Boaventura, M., and Nonato, L. G. (2014a). Combining anisotropic diffuison, transport equation and texture synthesis for inpainting textured images. Pattern Rec. Letters, 36:36-45.

Casaca, W. and Boaventura, M. (2010). A decomposition and noise removal method combining diffusion equation and wave atoms for textured images. Mathematical Problems in Engineering, 2010:1-21.

Casaca, W., Boaventura, M., and de Almeida, M. P. (2011a). Classifying texture and free-textured content from images using nonlinear anisotropic diffusion. In 5th Conf. Mathematical Analysis, pages 210-214.

Casaca, W., Colnago, M., and Nonato, L. G. (2015a). Interactive image colorization using laplacian coordinates. In 16th International Conf. on Computer Analysis of Images and Patterns (CAIP) (accepted).

Casaca, W., Gomez-Nieto, E., de O.L. Ferreira, C., Tavares, G., Pagliosa, P., Paulovich, F., Nonato, L. G., and Paiva, A. (2012). Colorization by multidimensional projection. In 25th SIBGRAPI, pages 32-38.

Casaca, W., Gomez-Nieto, E., Hartmann, I., Taubin, G., and Nonato, L. G. (2015b). Dealing with multiple requirements in geometric arrangements. IEEE Trans. Vis. and Computer Graphics (TVCG) (accepted).

Casaca, W., Motta, D., Taubin, G., and Nonato, L. G. (2015c). A user-friendly interactive image inpainting framework using laplacian coordinates. In IEEE Int. Conference on Image Processing (ICIP) (accepted).

Casaca, W., Nonato, L. G., and Taubin, G. (2014b). Laplacian coordinates for seeded image segmentation. In IEEE Conference on Computer Vision and Pattern Recognition (CVPR), pages 384-391.

Casaca, W., Paiva, A., Gomez-Nieto, E., Joia, P., and Nonato, L. G. (2013b). Spectral image segmentation using image decomposition and inner product-based metric. J. of Math. Imag. and Vis., 45(3):227-238.

Casaca, W., Paiva, A., and Nonato, L. G. (2011b). Spectral segmentation using cartoon-texture decomposition and inner product-based metric. In 24th SIBGRAPI, pages 266-273.

Chung, F. (1997). Spectral Graph Theory. CBMS, Ameciran Mathematical Society.

Couprie, C., Grady, L., Najman, L., and Talbot, H. (2011). Power watershed: A unifying graph-based optimization framework. IEEE Trans. on PAMI, 33(7):1384-1399.

Cour, T., Bénézit, F., and Shi, J. (2005). Spectral segmentation with multiscale graph decomposition. In IEEE Conference on Computer Vision and Pattern Recognition (CVPR), pages 1124-1131.

Cousty, J., Bertrand, G., Najman, L., and Couprie, M. (2009). Watershed cuts: Minimum spanning forests and the drop of water principle. IEEE Trans. on PAMI, 31(8):1362-1374.

Gomez-Nieto, E., Casaca, W., Nonato, L. G., and Taubin, G. (2013). Mixed integer optimization for layout arrangement. In 26th Conference on Graphics, Patterns and Images (SIBGRAPI), pages 115-122.

Gomez-Nieto, E., Roman, F., Pagliosa, P., Casaca, W., Helou, E., Oliveira, M. C., and Nonato, L. G. (2014). Similarity preserving snippet-based visualization of web search results. IEEE TVCG, 20:457-470.

Grady, L. (2006). Random walks for image segmentation. IEEE Trans. on PAMI, 28(11):1768-1783.

Joia, P., Gomez-Nieto, E., Neto, J. B., Casaca, W., , Paiva, A., and Nonato, L. G. (2012). Class-specific metrics for multidimensional data projection applied to cbir. Visual Computer, 28(10):1027-1037.

Peng, B. and Zhang, D. (2013). A survey of graph approaches to image seg. Pattern Rec., 46(3):1020-1038.

Shi, J. and Malik, J. (2000). Normalized cuts and image segmentation. IEEE Trans. on PAMI, 22:888-905. 